\title{
A synthetic predator odor (TMT) enhances conditioned analgesia and fear when paired with a benzodiazepine receptor inverse agonist (FG-7142)
}

\author{
GREGORY HOTSENPILLER and JON L. WILLIAMS \\ Kenyon College, Gambier, Ohio
}

\begin{abstract}
Benzodiazepine receptor inverse agonists, such as B-carboline-3-carboxylic acid n-methylamide (FG-7142), induce a state of fear and analgesia. These reactions can be conditioned to novel stimuli during drug administration. The present experiment was conducted to determine if FG-7142 is similar to other stressors such that certain ethologically relevant stimuli are more likely to become conditioned to a state of fear than are others. 2,5-Dihydro-2,4,5-trimethylthiazoline (TMT), a compound previously isolated from the feces of the predator fox, and butyric acid (BA) were presented to male rats as novel odors after administration of FG-7142 or vehicle. A method of ensuring that equal amounts of these odors were presented to the subjects was employed. Six hours after one conditioning trial with drug or vehicle, the same nonodor or chemical odors were present during formalin tests for analgesia and fear-induced freezing. Compared with no-odor and BA groups, the TMT group showed significantly enhanced conditioned analgesia and fear when FG-7142 was administered during the conditioning session. However, relative to the other odor conditions, the TMT condition produced significantly higher levels of analgesia and freezing in the absence of the benzodiazepine inverse agonist, suggesting that this odor acts as an unconditioned stressor by itself.
\end{abstract}

Benzodiazepine inverse agonists, such as FG-7142, fit the benzodiazepine receptor site but do not produce the allosteric effects that increase the binding of the neurotransmitter gamma-aminobutyric acid (GABA). Instead, the inverse agonists have potent intrinsic effects opposite to those of the benzodiazepine agonists (Crawley et al., 1985; Insel et al., 1984). Experimentation with human volunteers has shown that inverse agonists create anxiety symptoms, such as increased heart rate, palpitations, restlessness, and a feeling of impending doom (Dorrow, Horowski, Paschelke, Amin, \& Braestrup, 1983). Research involving primates has demonstrated similar physiological and behavioral findings (Crawley et al., 1985; Ninan et al., 1982).

The discovery of benzodiazepine inverse agonists having opposing pharmacological characteristics to fearreducing benzodiazepine agonists motivated experimenters to determine the relationship between such a chemically induced fear and other experimental methods of inducing fear, typically involving nociceptive stimuli. For instance, both systemic and ventricular administration of benzodiazepine inverse agonists produced a learned-helplessness

This research was partially supported by endowment funds from the Samuel B. Cummings Professorship in Psychology awarded to J.L.W. The authors are grateful to Dudley G. Thomas of the Kenyon College Department of Chemistry and Jennifer V. Hines of the Ohio State University College of Pharmacy for their advice and assistance with odor measurement and drug preparation. Correspondence should be addressed to J. L. Williams, Department of Psychology, 326 Samuel Mather Bldg., Kenyon College, Gambier, OH 43022 (e-mail: williams@ kenyon.edu) effect equivalent to one session of inescapable shock (Drugan, Maier, Skolnick, Paul, \& Crawley, 1985; Maier, Busch, Maswood, Grahn, \& Watkins, 1995). Furthermore, administering benzodiazepine inverse agonists, both in vivo and in vitro, has been found to decrease the density of rat GABA receptors, as does footshock exposure (Biggio, Concas, Mele, \& Corda, 1987).

The ability to condition stimuli, present during an induced-fear state from the administration of a benzodiazepine inverse agonist, has been demonstrated using the place-conditioning paradigm (Tsuda, Ida, Nishimura, \& Tanaka, 1989). Subjects administered a benzodiazepine inverse agonist during training spent a significantly longer amount of time in the opposing side of a maze during testing. Therefore, following the inverse agonist administration, it was assumed that fear was conditioned to the contextual cues of that particular side, which resulted in anxiety and, hence, avoidance of that side during later testing.

The experience of an aversive event is also known to result in analgesia, or a reduction in the perception and/or reactivity to pain (see Maier, 1986, for a review of this literature). The simultaneous display of fear and analgesia suggests that both can be conditioned to the same stimuli during exposure to a stressor, which has been demonstrated in studies using shock (Fanselow, 1984; Fanselow $\&$ Baackes, 1982) and social defeat by a conspecific (Hotsenpiller \& Williams, 1996; Williams, Just, \& Worland, 1994; Williams, Worland, \& Smith, 1990). Just as the benzodiazepine inverse agonists mimic the behavioral effects 
of aversive stressors, they also produce analgesia (Biggio et al., 1987; Helmstetter, Calcagnetti, \& Fanselow, 1990), and this analgesia can be conditioned to previously neutral stimuli (Fanselow \& Kim, 1992).

Considerable evidence indicates that certain stimuli can be more readily conditioned to fear-eliciting stimuli than can others. For example, when cat odors were associated with inescapable shock, as opposed to the hedonically neutral odor of citronella, more freezing was found during a subsequent test involving a single shock from a wall prod in the presence of cat odors (Williams \& Scott, 1989). This striking increase in aversion to cat odors was not interpreted to be solely due to an innate fear, but it was also speculated to be because of a selective ability of such predator odors to be very effective conditioned stimuli, perhaps as a result of some form of "biological preparedness or relevance." For example, Williams and Groux (1993) demonstrated that different odors had distinct effects when presented with a stressor. After baseline testing of odor preference, rats that received inescapable shock subsequently reduced their preferences for the odors of shocked conspecifics and predator cat odors but increased their preference for non-alpha conspecific odors. Therefore, the experience of fear differentially altered the relative preference for various naturalistic odors.

Although the administration of a benzodiazepine inverse agonist mimics inescapable shock, in that it produces fear and analgesia that can be conditioned to contextual stimuli, the degree to which such an inverse agonist might be selectively associated with various cues is not known. The potential for benzodiazepine-inverse-agonistinduced fear and analgesia to be differentially conditioned would demonstrate that such learning is not simply dependent upon stimulus contingencies, but it might also be a result of the organism having a unique ability to associate certain conditioned stimuli with an internal state of fear that is independent of painful stimulation.

One of the purposes of the present experiment was to examine whether or not certain conditioned stimulus odors (CSs), which were paired with an internal state of fear induced by FG-7142 (US), would produce subsequent conditioned fear and analgesia responses (CRs) to the same CS odors $6 \mathrm{~h}$ later. To ascertain whether certain CSs might produce greater fear-mediated reactions because of a unique association between the $\mathrm{CS}$ and the US requires that the putative CSs be equivalent in terms of their intensity or salience. That is, it is possible that an increase in the conditioning of fear and analgesia, upon the presentation of a certain odor, could simply be because that odor was stronger or more intense than were other odors at the time of conditioning and/or testing. If this were the case, it obviously could not be concluded that such a CS odor had a greater intrinsic value to be associated with a state of fear because of its biological or ethological significance.

One of the unique aspects of the present study was that it involved testing a putative $\mathrm{CS}$ that was a synthetic compound originally obtained from the droppings of a natural predator of the rat. Since the molecular weight of this compound was known, it was possible to determine the exact amount of this chemical to be used in order to have it be equivalent to another, control odor of a known molecular weight. In this way, the potential CS odors would be the same in terms of the number of odorous molecules emitted for a given concentration per unit time. Obviously, this method cannot be used with soiled cat bedding or other previously used natural odors (e.g., a mixture of urine, feces, hair, etc.), since their molecules and concentrations cannot be precisely determined.

2,5-Dihydro-2,4,5-trimethylthiazoline (TMT) is a compound that has been isolated from fox (Vulpes vulpes) (Vernet-Maury, 1980). This compound has been found to produce equivalent fear responses as whole fecal extracts of fox in terms of suppressing open-field activity and increasing corticosterone levels in rats (Vernet-Maury, Polak, \& Demael, 1984). Furthermore, it has been speculated that these reactions are innate; rat pups responded in a fear-like manner from birth to adulthood, without evidence of habituation of freezing during open-field tests (Vernet-Maury, Constant, Chanel, 1992).

Although TMT and fox droppings appear to be equally aversive to rats, the use of control odors in this experiment was critical because the introduction of any odor, whether natural or not, can produce a neophobic reaction. For instance, mice showed increased avoidance and analgesia reactions to cinnamon as well as predator odors (Kemble \& Gibson, 1992), and chocolate induces many of the same risk-assessment behaviors as do feared mammalian odors (Garbe, Kemble, \& Rawleigh, 1993). Relative to a high concentration of butyric acid as a control odor, Robinson (1990) found avoidance reactions to a predator odor to be stronger, suggesting that this aversion was more than a neophobic and/or intensity effect. However, in all of the previously mentioned studies, researchers have failed to equalize the intensity of the CS odors during classical conditioning. Therefore, while controlling for both the novelty and intensity of odors, the present experiment involved the conditioning of fear reactions resulting from FG-7142 administration in the presence of the predator odor of TMT, the novel odor of butyric acid, and a no-specific-odor control condition.

\section{METHOD}

\section{Subjects}

The subjects were 60 experimentally naive male rats, approximately $350 \mathrm{~g}$, bred in the Kenyon College Psychology Department Laboratory from Sprague-Dawley (Harlan) descent. Two rats were maintained in each cage, with food and water available ad lib, until 1 week before manipulation, at which time the rats were placed in separate cages in the same room. All experimental manipulations occurred in the light phase of a 12:12-h light:dark schedule.

\section{Apparatus}

Habituation, conditioning, and testing occurred in $36 \times 36 \times$ $41 \mathrm{~cm}$ metal boxes. A clear, ventilated, Plexiglas lid was affixed to the top of these boxes, and an 8-cm layer of corn-cob bedding ("Bed-O-Cobs") was spread over the bottom of the boxes. A 9.0$\mathrm{cm}$ Whatman \#1 filter paper (qualitative, medium-fast filter speed, medium crystalline retention, $0.06 \%$ ash; W\&R Baaston Limited, England) was hung with fishing line (Trilene, $8-\mathrm{lb}$ test) $3 \mathrm{~cm}$ from 
the ventilation lid for presentation of odor or no odor, depending on the group. A Panasonic WV-3260 video camera, mounted above the experimental boxes, was used to record the behavior of the subjects during testing. A white-noise generator was used during habituation, conditioning, and testing; it produced a $75 \mathrm{~dB}$ SPL background noise to prevent extraneous sounds from distracting the subjects.

\section{Odors}

2,5-Dihydro-2,4,5-trimethylthiazoline (TMT) was purchased from Phero Tech Inc. (British Columbia, Canada) as a neat liquid with a molecular weight of 129 . Butyric acid was purchased from Sigma Chemical Co. (St. Louis, MO) as a neat liquid with a molecular weight of 90.13 .

Pilot studies were conducted to establish criteria for ensuring equal amounts of these compounds to the subjects. Neat liquid was placed on a filter paper hung $3 \mathrm{~cm}$ from the top of the chamber. At the bottom of this chamber, on top of the $3 \mathrm{~cm}$ of bedding material, was placed a petri dish (Falcon 1029) with $10 \mathrm{~g}$ of activated charcoal ( 400 mesh; Norit), weighed before and after the application of odor. A nonodor condition, in which there was no liquid odor placement, was done prior to each odor condition to establish a proper baseline of absorbance of the charcoal in the absence of any applied odor. Such a control allowed for the added weight gain of the odor condition, relative to the nonodor condition, to be considered as a result of the absorbance of the particular compound. Therefore, after the weight gain due to the compound was determined, dividing this by the molecular weight of each compound yielded the approximate number of molecules absorbed during that period of time.

When a range of approximately $35-210 \mu \mathrm{l}$ was placed on the filter paper (via 9-in. glass pasteur pipet where 1 drop administered approximately $35 \mu \mathrm{l}$ ) for $30 \mathrm{~min}$, at each of three levels measured, the activated charcoal absorbed at least three times the number of molecules of TMT as it did butyric acid. Therefore, the amounts of the odors used in the actual experiment were approximately $35 \mu \mathrm{l}$ of TMT (average of $3.47^{19}$ molecules absorbed) and $105 \mu \mathrm{l}$ of butyric acid (average of $3.50^{19}$ molecules absorbed), since this was the lowest tested and observed demonstration of the $3: 1$ relationship of absorbance of TMT:butyric acid. Furthermore, this relationship of three times the amount of molecules of TMT being absorbed, relative to that of butyric acid was found to remain for at least $4 \mathrm{~h}$ (Hotsenpiller, 1995). Although less precise, glass pasteur pipets were used to administer odors to prevent absorption of the compounds into plastic pipet tips that fit pipetors. It should be noted that, at the amounts chosen for experimentation, TMT was hardly detectable to the experimenters, whereas the odor of butyric acid was quite intense.

\section{Drug}

B-carboline-3-carboxylic acid n-methylamide (FG-7142) was purchased from Sigma Chemical Co. (St. Louis, MO). This specific B-carboline was chosen because it is more metabolically stable than are others (Dorrow et al., 1983; Peterson, Maier, \& Seligman, 1993), and it is less likely to induce seizures than are other B-carbolines (Leidenheimer \& Schechter, 1988).

As with prior studies, Tween 80 was used to suspend the neat compound (Holmes \& Drugan, 1991; Leidenheimer \& Schechter, 1988; Meng \& Drugan, 1993; Tsuda et al., 1989). To each milliliter of distilled water used to dissolve the FG-7142 a drop of Tween 80 was added, and suspension was achieved with ultrasonic dispersion. Due to the rapid half-life of the B-carbolines (Mendelson, Cain, Cook, Paul, \& Skolnick, 1982; Schweri et al., 1983), $5 \mathrm{mg} / \mathrm{kg}$ of this solution was administered intraperitoneally (i.p.) immediately prior to the conditioning session. An equal volume of physiological saline was injected i.p. as a control.

The dosage of $5 \mathrm{mg} / \mathrm{kg}$ of FG-7142 was chosen after careful review of the literature. This dose has been demonstrated to be su- perior to lower doses in discrimination stability (Leidenheimer \& Schechter, 1988). It is also sufficient for decreasing open-field activity (Meng \& Drugan, 1993) and increasing avoidance (Holmes \& Drugan, 1991), and this dose is free of acute or chronic effects on locomotion (Brett, Jedrusik, Laverty, \& Pratt, 1995). Therefore, a single dose of $5 \mathrm{mg} / \mathrm{kg}$ was chosen because it was known to be sufficient for producing fear reactions and conditioning of fear, yet it was free from problems of seizures and motoric dysfunctions found with higher doses of this drug. Furthermore, some effects of B-carbolines are not always dose-related, and higher doses might induce a preconvulsive state that is likely to be different from the anxiogenic properties of these compounds (Thiebot, Soubrie, \& Sanger, 1988).

\section{Procedure}

In order to habituate to the experimental conditions, the subjects were placed in odor chambers with bedding, filter paper, dim light, and white noise, but no experimental odors were present at this time. The habituation sessions occurred twice daily for $30 \mathrm{~min}$ for 2 consecutive days. Following this, the subjects were randomly assigned to the six cells of a 2 (drug) $\times 3$ (odor) factorial design: saline administered with no odor during conditioning ( $\mathrm{SAL} / \mathrm{NO}$ ), saline administered with butyric acid odor (SAL/BA), saline administered with TMT odor (SAL/TMT), FG-7142 administered with no odor ( $\mathrm{FG} / \mathrm{NO}$ ), FG-7142 administered with butyric acid (FG/BA), and FG-7142 administered with TMT (FG/TMT).

On the day following the 2 nd habituation day, a single conditioning session was given. The subjects were injected with FG-7142 or saline and immediately placed in chambers with the respective odor added to the filter paper, with the same dim light on, and with the white noise that was present during habituation. The conditioning session lasted $30 \mathrm{~min}$, after which the subjects were returned to their home cages. The bedding material was changed before the next subject was run. New filter paper and odors were added to the chamber after every 5 th subject, which was approximately $3 \mathrm{~h}$. This was done, rather than adding additional odors after each subject, in order to avoid a possible ceiling effect of fear and analgesia due to the accumulation of odors. Furthermore, the method for the determination of odor absorption was not conducted under conditions of repeated addition of the various odors. The interval chosen for additional odor administration seemed reasonable considering that the $3: 1$ relationship of TMT:BA absorbance was still evident after $4 \mathrm{~h}$.

Six hours following the single conditioning sessions, the subjects were given a $50-\mu \mathrm{l}$ injection of $15 \%$ formalin solution under the dorsal surface of the right rear paw and returned to their home cages for $15 \mathrm{~min}$. Then, the subjects were tested with the same odor that was present during the conditioning session, using the same order, with clean bedding, and fresh odor added, as described previously. A Panasonic WV-3260 video camera, mounted above the chamber, was used to record the behavior of the subjects. During the 16-min test session, an 8-sec time-sampling procedure was used, and tabulations were made of the following responses: (1) freezing, a crouched posture with no movement except for respiration; (2) paw licking, grasping and licking the formalin-injected paw while lying down; and (3) general activity, all other behaviors. The formalin concentration, drug absorption time, time of testing, and the time-sampling procedure have been extensively used by others (Fanselow, 1984; Fanselow \& Helmstetter, 1988; Hotsenpiller \& Williams, 1996; Williams et al., 1994; Williams et al., 1990).

\section{RESULTS}

The results of the paw licking of the six groups during the formalin test are depicted in Figure 1. A $2 \times 3$ independent-groups analysis of variance (ANOVA) of the paw-licking data revealed main effects for drug con- 


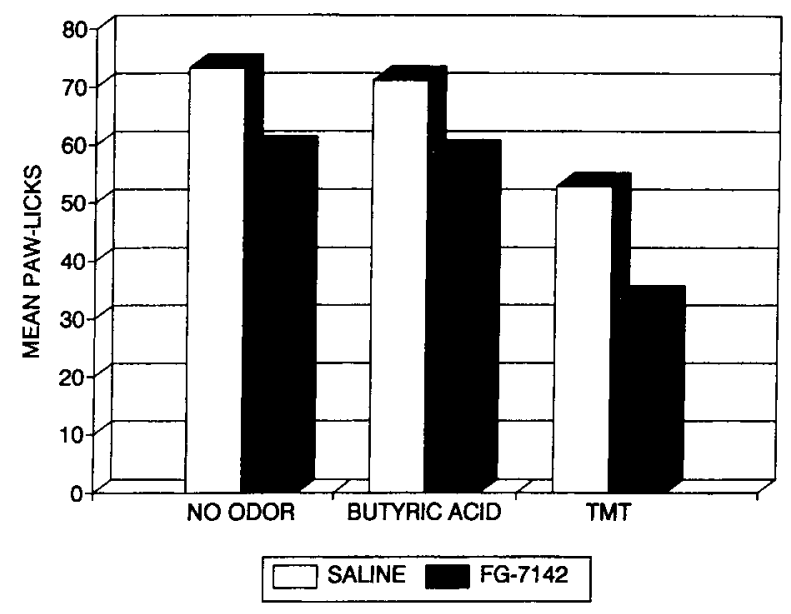

Figure 1. Mean number of observations of paw licking during formalin tests. Tests occurred $6 \mathrm{~h}$ after conditioning either with saline or with FG-7142 and the corresponding odor type. Odors were present during testing as conditioned stimuli. No drugs were administered during the test session.

dition $[F(1,58)=21.19, p<.001]$ and type of odor during conditioning/testing $[F(2,57)=19.86, p<.001]$. No significant interaction between the drug and odor conditions was found.

Post hoc analysis, using Tukey's tests, showed that the number of recorded paw-licking instances for each odor condition was lower in the FG-7142 conditions, suggesting increased conditioned analgesia in these groups (for the NO and BA groups, $p \mathrm{~s}<.05$; for the TMT group, $p<$ $.01)$. It should be noted that, within the odor conditions, differences between saline and FG-7142 were greater in the TMT condition than in the two other odor conditions.

The SAL/TMT group showed significantly lower levels of paw licking than did the SAL/BA and SAL/NO groups $(p s<.01)$. The FG/TMT group displayed significantly less paw-licking than did the other FG groups in the BA and NO conditions ( $p s<.01)$. The TMT effects in the SAL and FG groups are in contrast to those in the BA groups: BA subjects showed paw licking similar to that of the subjects in the respective NO groups.

The results of the freezing data obtained from formalin tests are presented in Figure 2 . A $2 \times 3$ ANOVA revealed a main effect for both drug type $[F(1,58)=20.16, p<$ $.001]$ and odor condition $[F(2,57)=11.33, p<.001]$. Consistent with the paw-licking data, each odor-FG-7142 condition showed increased freezing as a conditioned response (for NO groups, $p<.01$; for BA and TMT groups, $p s<.05$ ). As with the paw-licking data, no significant interaction between drug and odor conditions was found.

Post hoc Tukey's analysis of the same drug conditions revealed that only the two TMT groups differed from the two NO groups. The SAL/TMT group displayed more freezing than did the SAL/NO group $(p<.01)$. The FG/ TMT group showed more freezing than did the FG/NO group $(p<.01)$. In contrast to the paw-licking data, the same SAL and FG groups that had TMT did not differ from either of the two respective BA groups. However, the FG/TMT group showed the highest level of freezing, relative to the two other conditioned FG groups.

\section{DISCUSSION}

FG-7142 augmented conditioned fear reactions as evidenced by both increased analgesia and increased freezing during later odor tests, without this anxiogenic drug. Such results are not surprising considering the previous research demonstrating the ability of benzodiazepine inverse agonists to mimic the effects of inescapable shock as a stressor (Drugan et al., 1985; Duka \& Stephens, 1986; Maier et al., 1995), to produce fear-like responses (Biggio et al., 1987; Fanselow \& Kim, 1992; Holmes \& Drugan, 1991; Meng \& Drugan, 1993), and to result in conditioning with previously neutral stimuli (Helmstetter et al., 1990; Tsuda et al., 1989).

In addition, the increase in conditioned fear-related behaviors displayed by the TMT groups is not surprising considering that this odor has been demonstrated to increase corticosterone levels while decreasing open-field activity (Vernet-Maury et al., 1984) and to increase freezing (Vernet-Maury et al., 1992). However, the results of the present study are unique in that they clearly indicate that the predator odor of TMT, by itself, produces stressinduced analgesia and freezing. The argument that stimulus intensity or novelty is responsible for the powerful effects of TMT is untenable, because not only was butyric acid used as a control odor but these two compounds were used in amounts that produced a comparable number of molecules absorbed by activated charcoal.

Previous studies have demonstrated the ability of certain stimuli to enhance the strength or rate of conditioned fear reactions (Mineka \& Cook, 1988; Williams \& Scott, 1989). Such an ability for an organism to be selective or

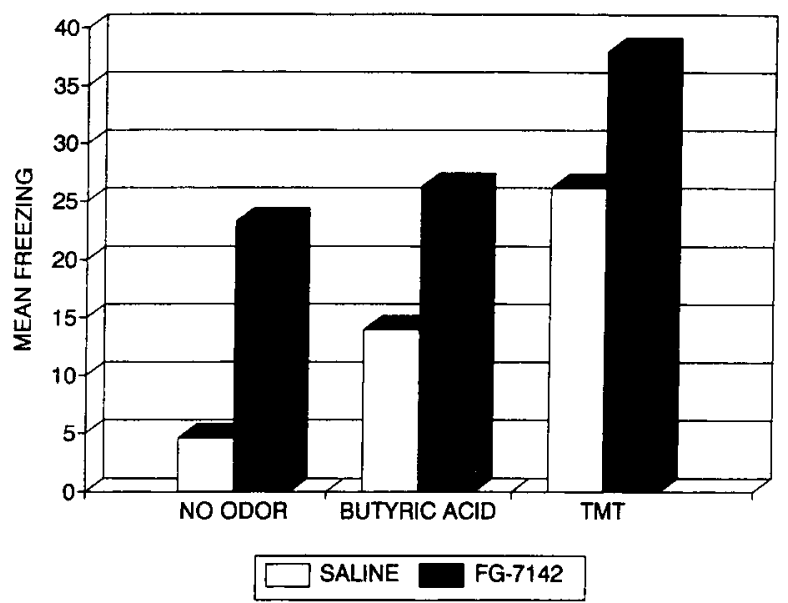

Figure 2. Mean number of observations of freezing during formalin tests. Tests occurred $6 \mathrm{~h}$ after conditioning either with saline or with FG-7142 and the corresponding odor type. Odors were present during testing as conditioned stimuli. No drugs were administered during the test session. 
to have a biological preparedness to associate certain conditioned stimuli with aversive events can be thought of as being advantageous in terms of evolution. Although the combination of TMT as a CS and FG-7142 as a stressor (US) resulted in the greatest amount of analgesia and freezing, these effects were found to be additive because the interaction between the odor and drug conditions was not found to be statistically significant. Therefore, although the high degree to which the rats were able to associate the isolated odor from fox droppings with a state of fear allows for evolutionary speculation, the argument of biological preparedness was not fully supported by these data. Furthermore, the lack of support for a unique association between TMT and FG-7142 does not rule out the possibility that a relationship between a predator odor and stress indeed exits. Multiple stressors must be used in conjunction with this novel predator odor to determine whether a certain type of stressor (e.g., shock, social defeat by a dominant conspecific) is required. However, it would be difficult to equate the intensity of the different stressors in this type of research. Therefore, the results of the present study indicate only that no selective associability exists with this predator odor and a chemically induced fear (via FG-7142). Regardless of this issue, the fact that the odor of TMT was found to elicit conditioned and unconditioned fear-related reactions, in the present study, has interesting implications concerning the ethopsychopharmacology of the rat's defense systems, which definitely need to be addressed in future research.

Despite the fact that the amount of odors applied to the filter paper was quantified and then equated in terms of the number of molecules absorbed by activated charcoal, an argument could be made that what binds to the activated charcoal does not necessarily bind in the same quantity and/or affinity to the nasal membrane of the rat. Although valid, this point does not directly apply to the present results because activated charcoal was used in an effort to equalize the number of molecules available to the rat itself, not the binding characteristics of the nasal membrane. In addition, TMT may elicit a more potent and/or unique response than does butyric acid. Such differences could be measured with electrophysiological techniques, but these, again, are beyond the scope and thrust of the present experiment, which was done solely to ensure equal amounts of availability of the odor sources to the subjects. Furthermore, it is believed that odor novelty and salience cannot account for the reported findings, because three times the amount of butyric acid, relative to TMT, was applied to the filter paper, and the butyric acid was perceived as being much more intense by the experimenters.

The results of this experiment extend beyond that of testing for selective or differential CS-US conditioning. The barely perceptible odor of TMT, which produced the strongest fear reactions in this experiment, demonstrates the need for proper control groups and the inability to rely on human senses to control for CS variables or effects during odor conditioning experiments using animals as sub- jects. In addition, the findings of this experiment emphasize the importance of measuring more than one response in order to gain a greater understanding of the nature of fear conditioning. The use of different measures of behavior are particularly important when freezing is evaluated, because this response has sometimes been shown to be independent of other fear-mediated reactions (Williams \& Scott, 1989). Clearly, the present results support the notion that benzodiazepine inverse agonists and synthetic predator odors function comparably to other pain-inducing stressors, such as shock or defeat by a conspecific. Finally, the benefits of using an anxiogenic drug and/or a synthetic odor to examine various reactions to stress/ anxiety exposure are that they do not involve the consequences of physically painful stimuli, and both of these compounds can be precisely quantified and manipulated.

\section{REFERENCES}

Biggio, G., Concas, A., Mele, S., \& Corda, M. G. (1987). Changes in GABAergic transmission induced by stress, anxiogenic and anxiolytic B-carbolines. Brain Research Bulletin, 19, 301-308.

Brett, R. R., Jedrusik, P., Laverty, W., \& Pratt, J. A. (1995). Continuous exposure to $F G$ 7142: Behavioral sensitization is not accompanied by changes in benzodiazepine/GABA receptor coupling. Journal of Psychopharmacology, 9, 223-227.

Crawley, J. N., Ninan, P. T., Pickar, D., Chrousos, G. P., LinNolla, M., Skolnick, P., \& Paul, S. M. (1985). Neuropharmacological antagonism of the beta-carboline-induced "anxiety" response in Rhesus monkeys. Journal of Neuroscience, 5, 477-485.

Dorrow, R., Horowski, R., Paschelke, G., Amin, M., \& BraeSTRUP, C. (1983). Severe anxiety induced by FG 7142 , a betacarboline ligand for benzodiazepine receptors. Lancet, 41, 98-99.

Drugan, R. C., Maier, S. F., Skolnick, P., Paul, S. M., \& Crawley, J. N. (1985). An anxiogenic benzodiazepine receptor ligand induces learned helplessness. European Journal of Pharmacology, 113, 453-457.

Duka, T., \& Stephens, D. N. (1986). Potentiation of the propunishment, but not the convulsant action of the B-carboline DMCM by naltrexone. Pharmacology, Biochemistry \& Behavior, 25, 595-598

FANSELOW, M. S. (1984). Shock-induced analgesia on the formalin test: Effects of shock severity, naloxone, hypophysectomy, and associative variables. Behavioral Neuroscience, 98, 79-95.

FANSELOW, M. S., \& BAACKES, M. P. (1982). Conditioned fear-induced opiate analgesia on the formalin test: Evidence for two aversive motivational systems. Learning \& Motivation, 13, 200-221.

Fanselow, M. S., \& Helmstetter, F. J. (1988). Conditioned analgesia, defensive freezing, and benzodiazepines. Behavioral Neuroscience, 102, 233-243.

FANSELOW, M. S., \& KIM, J. J. (1992). The benzodiazepine inverse agonist DMCM as an unconditional stimulus for fear-induced analgesia: Implications for the role of $\mathrm{GABA}_{\mathrm{A}}$ receptors in fear-related behavior. Behavioral Neuroscience, 106, 336-344.

Garbe, C. M., Kemble, E. D., \& Rawleigh, J. M. (1993). Novel odors evoke risk assessment and suppress appetitive behaviors in mice. Aggressive Behavior, 19, 447-454.

Helmstetter, F. J., Calcagnetti, D. J., \& Fanselow, M. S. (1990). The beta-carboline DMCM produces hypoalgesia after central administration. Psychobiology, 18, 293-297.

Holmes, P. V., \& Drugan, R. C. (1991). Differential effects of anxiogenic central and peripheral benzodiazepine receptor ligands in tests of learning and memory. Psychopharmacology, 104, 249-254.

HotSENPILLER, G. (1995). [Weight gain of activated charcoal as a result of proximal odors]. Unpublished raw data.

Hotsenpiller, G., \& Williams, J. L. (1996). Conditioned fear and analgesia to conspecific odors: Benzodiazepine and 5- $\mathrm{HT}_{1 \mathrm{~A}}$ agonists. Psychobiology, 24, 118-126. 
Insel, T. R., Ninan, P. T., Aloi, J., Jimerson, D. C., Skolnick, P., \& Paul, S. M. (1984). A benzodiazepine receptor-mediated model of anxiety: Studies in nonhuman primates and clinical implications. Archives of General Psychiatry, 41, 741-750.

Kemble, E. D., \& Gibson, B. M. (1992). Avoidance and hypoalgesia induced by novel odors in mice. Psychological Record, 42, 555-563.

Leidenheimer, N. J., \& SChechter, M. D. (1988). Discriminative stimulus control by the anxiogenic b-carboline FG 7142: Generalization to a physiological stressor. Pharmacology, Biochemistry \& Behavior, 30, 351-355.

MAIER, S. F. (1986). Stressor controllability and stress-induced analgesia. In D. D. Kelley (Ed.), Stress-induced analgesia (Annals of the New York Academy of Sciences, Vol. 467, pp. 55-71). New York: New York Academy of Sciences.

Maier, S. F., Busch, C. R., Maswood, S., Grahn, R. E., \& Watkins, L. R. (1995). The benzodiazepine receptor inverse agonist DMCM microinjected into the region of the dorsal raphe nucleus mimics the effects of inescapable shock. Society for Neuroscience Abstracts, 21, 464 .

Mendelson, W. B., Cain, M., Cook, J. M., Paul, S. M., \& SkolNICK, P. (1982). Do benzodiazepine receptors play a role in sleep regulation? Studies with the benzodiazepine antagonist, 3-hydroxymethyl-B-carboline (3-HOC). In E. Usdin (Ed.), Beta-carbolines and tetrahydroisoquinolines (pp. 253-261). New York: Alan R. Liss.

Meng, I. D., \& Drugan, R. C. (1993). Sex differences in open field behavior in response to the B-carboline FG 7142 in rats. Physiology \& Behavior, 54, 701-705.

MineKA, S., \& COOK, M. (1988). Social learning and the acquisition of snake fear in monkeys. In T. R. Zentall \& B. G. Galef, Jr. (Eds.), Social learning: Psychological and biological perspectives (pp. 5173). Hillsdale, NJ: Erlbaum.

Ninan, P. T., Insel, T. M., Cohen, R. M., Cook, J. M., Skolnick, P. \& Paul, S. M. (1982). Benzodiazepine receptor-mediated experimental "anxiety" in primates. Science, 218, 1332-1334.

Peterson; C., Maier, S. F., \& Seligman, M. E. P. (1993). Learned helplessness: A theory for the age of personal control. New York: Oxford University Press

Robinson, I. (1990). The effect of mink odour on rabbits and small mammals. In D. W. Macdonald, D. Muller-Schwarze, \& S. E.
Natynczuk (Eds.), Chemical signals in vertebrates (Vol. 5, pp. 566572). Oxford: Oxford University Press.

Schweri, M. M., Martin, J. V., Mendelson, W. B., Barrett, J. E., PAul, S. M., \& SKOLNICK, P. (1983). Pharmacokinetic and pharmacodynamic factors contributing to the convulsant action of betacarboline-3-carboxylic acid esters. Life Sciences, 33, 1505-1510.

Thiebot, M.-H., Soubrie, P., \& Sanger, D. (1988). Anxiogenic properties of beta-CCE and FG-7142: A review of promises and pitfalls. Psychopharmacology, 94, 452-463.

TsudA, A., IDA, Y., Nishimura, H., \& TANAKA, M. (1989). Anxiogenic effects of $\beta$-CCE as measured in two different conditioning paradigms. Psychobiology, 17, 202-206.

Vernet-Maury, E. (1980). Trimethylthiazoline in fox feces: A natural alarming substance for the rat. In H. van der Starre (Ed.), Olfaction and taste (Vol. 7, p. 407). Washington, DC: IRL.

Vernet-Maury, E., Constant, B., \& Chanel, J. (1992). Repellent effect of trimethylthiazoline in the wild rat Rattus norvegicus Berkenhout. In R. L. Doty \& D. Muller-Schwarze (Eds.), Chemical signals in vertebrates (Vol. 6., pp. 305-310). New York: Plenum.

Vernet-Maury, E., Polak, E. H., \& Demael, A. (1984). Structureactivity relationship of stress-inducing odorants in the rat. Journal of Chemical Ecology, 10, 1007-1018.

Williams, J. L., \& GROUX, M. L. (1993). Exposure to various stressors alters preferences for natural odors in rats (Rattus norvegicus). Journal of Comparative Psychology, 107, 39-47.

Williams, J. L., Just, J. M., \& Worland, P. D. (1994). Effect of repeated-defeat sessions as a colony intruder on subsequent hypoalgesia and feeding in the rat. Learning \& Motivation, 25, 152-174.

Williams, J. L., \& SCOTT, D. K. (1989). Influence of conspecific and predatory stressors and their associated odors on defensive burying and freezing responses. Animal Learning \& Behavior, 17, 383-393.

Williams, J. L., Worland, P. D., \& SMith, M. G. (1990). Defeatinduced hypoalgesia in the rat: Effects of conditioned odors, naltrexone, and extinction. Journal of Experimental Psychology: Animal Behavior Processes, 16, 345-357.

(Manuscript received July 18, 1996; revision accepted for publication September 26, 1996.) 